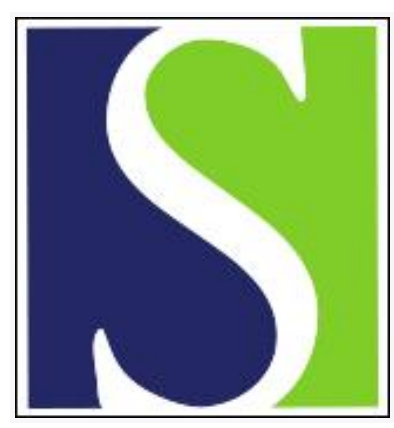

Scand J Work Environ Health 1999;25(3):278-284

https://doi.org/10.5271/sjweh.435

Issue date: Jun 1999

Magnitude of acute exposures to vibration and finger circulation

by Bovenzi M, Lindsell CJ, Griffin MJ

The following articles refer to this text: 2002;28(5):324-327;

2006;32(5):383-391

Key terms: acceleration magnitude; acute vibration; digital vasoconstriction; finger blood flow; vasomotor effect

This article in PubMed: www.ncbi.nlm.nih.gov/pubmed/10450780

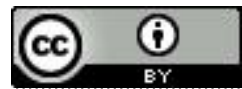




\title{
Magnitude of acute exposures to vibration and finger circulation
}

\author{
by Massimo Bovenzi, MD, ${ }^{1}$ Christopher J Lindsell, BEng, ${ }^{2}$ Michael J Griffin, PhD ${ }^{2}$
}

\begin{abstract}
Bovenzi M, Lindsell CJ, Griffin MJ. Magnitude of acute exposures to vibration and finger circulation. Scand $J$ Work Environ Health 1999;25(3):278-284.
\end{abstract}

\begin{abstract}
Objectives Changes in finger circulation were studied during and after acute exposure to increasing magnitudes of hand-transmitted vibration.

Methods Finger skin temperature (FST) and finger blood flow (FBF) were measured in the middle fingers of both hands of 10 healthy men. The right hand was exposed for 15 minutes to $125-\mathrm{Hz}$ vibration with acceleration magnitudes of either $5.5,22,44$, or $62 \mathrm{~m} / \mathrm{s}^{2}$ root-mean-square. The measures of finger circulation were taken before the vibration, at fixed intervals during exposure, and during a 45-minute recovery period.

Results The FST did not change during vibration exposure, whereas vibration of any magnitude provoked significant reductions in the FBF of the vibrated finger when compared with the preexposure FBF and the contralateral (nonvibrated finger) FBF. Vasoconstrictor aftereffects (ie, during recovery) were observed in both fingers after the end of exposure to vibration magnitudes greater than $22 \mathrm{~m} / \mathrm{s}^{2}$ root-mean-square. The higher the vibration magnitude, the stronger the reduction of FBF in either finger during both vibration exposure and the recovery period. This effect was stronger in the vibrated finger than in the nonvibrated finger during both periods. Conclusions Acute exposure to $125-\mathrm{Hz}$ vibration can reduce $\mathrm{FBF}$ in both the vibrated and the nonvibrated finger, and the degree of digital vasoconstriction is related to the magnitude of the vibration. The pattern of the hemodynamic changes during and after vibration exposure suggests that complex vasomotor mechanisms are involved in the response of digital vessels to acute vibration.
\end{abstract}

Key terms acceleration magnitude, acute vibration, digital vasoconstriction, finger blood flow, vasomotor effects.

In a previous paper, we reported that the duration of acute exposures to hand-transmitted vibration can influence the response of finger circulation in healthy persons (1). It was found that the longer the duration of exposure to vibration with a frequency of $125 \mathrm{~Hz}$ and a root-meansquare $(\mathrm{rms})$ acceleration magnitude of $87 \mathrm{~m} / \mathrm{s}^{2}$, the stronger the vasoconstriction in the vibrated finger during both vibration exposure and a subsequent recovery period. Current standards for hand-transmitted vibration assume that the risk of vascular disorders in the fingers and hands of vibration-exposed subjects depends primarily on the physical characteristics of vibration $(2,3)$. Thus, in addition to the duration of exposure, the magnitude and frequency of vibration have to be taken into account when the probability of adverse effects in the digital vessels is predicted.

The aim of this study was to extend our previous investigation by exploring the effect of the magnitude of vibration acceleration on the finger circulation of normal subjects. This study is part of a research project whose purpose is to contribute to the understanding of the patho physiological mechanisms underlying the digital circulatory responses to vibration and how they are related to the physical characteristics of vibration $(4,5)$.

\section{Subjects and methods}

\section{Subjects}

Ten healthy male volunteers, 6 Caucasians and 4 Orientals with a mean age of 32 (SD 8.5) years, participated in the study. All the subjects were office workers with no history of regular usage of vibrating tools in occupational or leisure-time activities. None of them reported cardiovascular or neurological disorders, connective

1 Institute of Occupational Medicine, University of Trieste, Trieste, Italy.

2 Human Factors Research Unit, Institute of Sound and Vibration Research, University of Southampton, Southampton, United Kingdom.

Reprint requests to: Dr Massimo Bovenzi, Istituto di Medicina del Lavoro, Università di Trieste, Centro Tumori, Via della Pietà, 19I-34129 Trieste, Italy. [E-mail: bovenzi@univ.trieste.it] 
tissue diseases, injuries to the upper extremities, or a family history of Raynaud's phenomenon. All were nonsmokers and reported mild alcohol consumption. All the subjects read a list of contraindications and gave written, informed consent to participate in the investigation. The mean height of the subjects was 176 (SD 5.0) cm, and their mean weight was 76 (SD 15) kg. Finger dimensions were measured with vernier callipers to a precision of $0.5 \mathrm{~mm}$. Finger volume and surface area were calculated from that of a cylinder formed from an ellipse based on the dimensions of the proximal interphalangeal joint and the length of the finger. The subjects were requested to avoid consuming caffeine for 2 hours and alcohol for 12 hours prior to the testing.

\section{Measures of finger circulation}

Finger blood flow (FBF) and finger systolic blood pressure (FSBP) were measured in the middle fingers of both the right and the left hand. The methods used to measure FBF and FSBP were similar to those reported elsewhere $(1,4,5)$. Briefly, mercury-in-silastic strain gauges were placed around the distal phalanges at the base of the nails, and plastic pressure cuffs for air inflation $(2.4 \times 9 \mathrm{~cm})$ were fixed around the proximal phalanges and secured with a Velcro strip. The pressure cuffs and strain gauges were connected to a plethysmograph (Digitmatic DM2000, Medimatic A/S, Copenhagen).

The FBF was measured using a venous occlusion technique. The pressure cuffs were inflated to a pressure of between 40 and $60 \mathrm{~mm} \mathrm{Hg}(5.3$ and $8.0 \mathrm{kPa})$, and the rise of volume was detected by means of the strain gauge according to the criteria given by Greenfield et al (6). The FBF measurements were expressed as milliliters per 100 milliliters a minute. Between 3 to 5 plethysmographic recordings of FBF were made for each measurement, and the mean value was calculated. Each plethysmographic recording lasted about 15 seconds.

The FSBP was measured using the technique described by Nielsen et al (7). The tips of the middle fingers were squeezed, and the pressure cuffs were inflated to a suprasystolic pressure of $250 \mathrm{~mm} \mathrm{Hg}(33.3 \mathrm{kPa})$. The pressure in the cuffs was then reduced at approximately $3 \mathrm{~mm} \mathrm{Hg} / \mathrm{s}(0.4 \mathrm{kPa} / \mathrm{s})$. The FSBP was defined as the cuff pressure at which a volume increase was detected in the distal phalanx by means of the strain gauge.

The brachial systolic and diastolic blood pressures were measured in the upper right arm using an ausculatory technique with a standard pressure cuff $(12 \times 23 \mathrm{~cm})$. The finger and brachial blood pressures were obtained at the beginning and at the end of each experimental session.

Finger skin temperature (FST) was measured using k-type thermocouples connected to a HVLab thermal esthesiometer so as to measure temperatures to an accuracy of $\pm 0.2^{\circ} \mathrm{C}$. The thermocouples were taped to the dorsal surface of the medial phalanges of the middle fingers using porous surgical tape so as to minimize the influence on skin temperature (Micropore, 3M). The room temperature was measured by a mercury-in-glass thermometer to an accuracy of $\pm 0.1^{\circ} \mathrm{C}$.

\section{Experimental procedure}

The experiments were performed in a room with a mean temperature of $24.5(\mathrm{SD} 0.8)^{\circ} \mathrm{C}$. The subjects wore light indoor clothing and lay supine on an examination couch throughout the investigation with their hands resting on wooden platforms alongside the body at about the level of the heart. After a period of acclimatization of about 15-20 minutes, FBF and FST were measured in the middle fingers of both hands. After the base-line readings were obtained, the subjects were asked to apply a downward force of $10 \mathrm{~N}$ with their right hand on a wooden platform $(100 \times 100 \mathrm{~mm})$, which was mounted on an electrodynamic vibrator (V404, Ling Dynamics). Visual feedback for the control of downward force was supplied by means of an analogue force meter connected to a force cell mounted between the platform and the shaker. All fingers of the right hand were in contact with the wooden platform (4). The right elbow and upper arm rested comfortably on the couch, which had no contact with the vibrating system. The arrangement for controlling contact force and generating vibration has been described elsewhere $(1,4,5)$.

Sinusoidal vibration was produced in the vertical direction at a frequency of $125 \mathrm{~Hz}$ and an rms acceleration of $5.5,22,44$, or $62 \mathrm{~m} / \mathrm{s}^{2}$ (unweighted). These values correspond to frequency-weighted accelerations of 0.7 , $2.8,5.5$, and $7.8 \mathrm{~m} / \mathrm{s}^{2} \mathrm{rms}$, respectively, according to the international standard ISO 5349 and the British standard BS $6842(2,3)$.

The exposure duration on each occasion was $15 \mathrm{~min}$ utes, giving 8-hour energy-equivalent frequency-weighted acceleration magnitudes of $0.12,0.5,1.0$, and $1.4 \mathrm{~m} / \mathrm{s}^{2} \mathrm{rms}$, respectively. According to the dose-response relationship included in an annex to BS 6842, vibration-induced white finger (VWF) is expected to occur in $10 \%$ of a worker population after about 16 years of exposure to an equivalent-weighted acceleration magnitude of $1.4 \mathrm{~m} / \mathrm{s}^{2}$, the highest magnitude used in this study (3).

The FBF and FST were measured from both the exposed (right) and unexposed (left) middle fingers before the vibration exposure, throughout the vibration exposure period, and for 45 minutes following the exposure. The measures of finger circulation were commenced at 0.5 , $1.5,3.5,5.5,7.5$, and 15 minutes after the start of vibration. The plethysmographic recordings showed no oscillations associated with the frequency of vibration. Measures were taken at the same intervals following the cessation of vibration and then at each 7.5-minute interval 
during the remainder of the recovery period. Previous studies have shown that these dependent variables (FBF and FST) do not change when the static force used in this experiment is applied without vibration $(1,4,5)$. For this reason, the present study does not include a static condition.

Each subject had measurements taken on 4 occasions and the right hand was exposed to each vibration magnitude on a different day. The order of presentation of vibration magnitude was randomized. Each experimental session lasted about 1.5 hours.

The study was approved by the Human Experimentation Safety and Ethics Committee of the Institute of Sound and Vibration Research at the University of Southampton.

\section{Statistical methods}

The data analysis was performed using the software packages BMDP/Dynamic (release 7.0) and Stata (version 5.0). The data were summarized with the mean as a measure of central tendency and the standard deviation (SD) or standard error of the mean (SEM) as measures of dispersion. The difference between 2 means was tested by a paired or unpaired Student t-test when appropriate. A repeated measures analysis of variance (ANOVA) was used to test the hypothesis of no difference in the vascular responses under different exposure conditions ("treatments"). When the treatment-by-time interaction term was significant $(\mathrm{P}<0.05)$ in the ANOVA, a separate analysis of the results within treatments was made. To control for the effect of covariates on the response variables, a repeated measures analysis of covariance (ANCOVA) was also used. The $95 \%$ Bonferroni confidence intervals for pairwise mean comparisons of the response by time were used when the probability value for the F-test of the repeated measures ANOVA was $\mathrm{P}<0.05$ (2-sided). A regression analysis was performed by the method of the least squares. The relation between the FBF and vibration magnitude was assessed by the generalized estimating equations (GEE) approach to repeated measures data sets to account for the within-subject correlation (8).

\section{Results}

The vascular measurements taken before the vibration exposure showed neither changes in FBF, FST, or FSBP across the 4 experimental sessions nor differences between the middle-right and middle-left fingers within any sessions.

Under the preexposure conditions, the FBF correlated with the FST $(P=0.02)$ and finger volume ( $P=0.03)$ for both fingers. No differences were observed for the finger and brachial systolic blood pressures measured at the beginning and end of the testing sessions.
The environmental conditions were well controlled during the experimental sessions, and the repeated measures ANOVA showed no differences in room temperature across the 4 testing sessions, ranges of the mean values being $24.2(\mathrm{SD} 0.9)-24.9(\mathrm{SD} 0.7)^{\circ} \mathrm{C}$.

\section{Finger skin temperature}

Before the exposure, the mean FST values varied from $32.6\left(\mathrm{SD} 2.5\right.$ ) to $33.6(\mathrm{SD} 1.3)^{\circ} \mathrm{C}$ for the right (vibrated) finger and from 32.4 (SD 2.2) to 33.5 (SD 1.3) ${ }^{\circ} \mathrm{C}$ for the left (nonvibrated) finger. With control for age, finger surface area, and room temperature, the repeated measures ANCOVA showed neither treatment-by-time interaction nor differences in the FST between the 4 exposure conditions for either the vibrated or the nonvibrated finger during either the vibration exposure or the recovery period (results not shown).

\section{Finger blood flow}

Table 1 presents the results of the FBF measurements before, during, and after the exposures to vibration at 4 acceleration magnitudes. The FBF means of both the vibrated and nonvibrated fingers throughout the various experimental sessions are plotted in figure 1. As the repeated measures ANCOVA showed a treatment-by-time interaction for both the vibrated $(\mathrm{P}=0.001)$ and the nonvibrated $(\mathrm{P}=0.02)$ fingers, the changes in FBF were examined separately for the different exposure conditions.

Acute exposure to $125-\mathrm{Hz}$ vibration provoked significant changes in the FBF of the vibrated finger $(\mathrm{P}=0.001)$ with any acceleration magnitude. Compared with the preexposure measurements, a reduction in the FBF of the vibrated finger was observed in all the measurements during the exposure to vibration (Bonferroni test: $\mathrm{P}<0.05$ ). Moreover, the greater the vibration magnitude, the stronger the vasoconstriction in the vibrated finger from 1.5 to 15 minutes during the vibration exposure $(\mathrm{P}=0.01)$. Within each exposure condition, there was no difference between the consecutive measurements of the FBF (ie, the degree of vasoconstriction in the vibrated finger did not vary significantly throughout the 15-minute exposure period).

In the nonvibrated finger, vibration with acceleration magnitudes from 22 to $62 \mathrm{~m} / \mathrm{s}^{2}$ caused a decrease in the FBF $(\mathrm{P}=0.001)$, while no changes were observed during exposure to vibration acceleration of $5.5 \mathrm{~m} / \mathrm{s}^{2}$.

At each measurement time during the various exposure conditions, the reduction of FBF was greater in the vibrated finger than in the nonvibrated finger $(\mathrm{P}<0.05)$.

Immediately after the vibration ended, there was an increase in the FBF of both fingers. When compared with the last measure of FBF taken at the 15 th minute during the vibration exposure, the increase in FBF was significant for acceleration magnitudes of 22 to $62 \mathrm{~m} / \mathrm{s}^{2}$ for the vibrated finger $(\mathrm{P}=0.01)$ and for the acceleration magni- 
Table 1. Finger blood flow [ml/(100 ml-min)] measured in 10 healthy subjects before, during, and after 15-minute exposure to vibration with a frequency of $125 \mathrm{~Hz}$ and acceleration magnitudes of $5.5,22,44$, and $62 \mathrm{~m} / \mathrm{s}^{2} \mathrm{rms}$. (rms = root-mean-square, $\mathrm{SE}=$ standard error of mean, ANCOVA = repeated measures analysis of covariance)

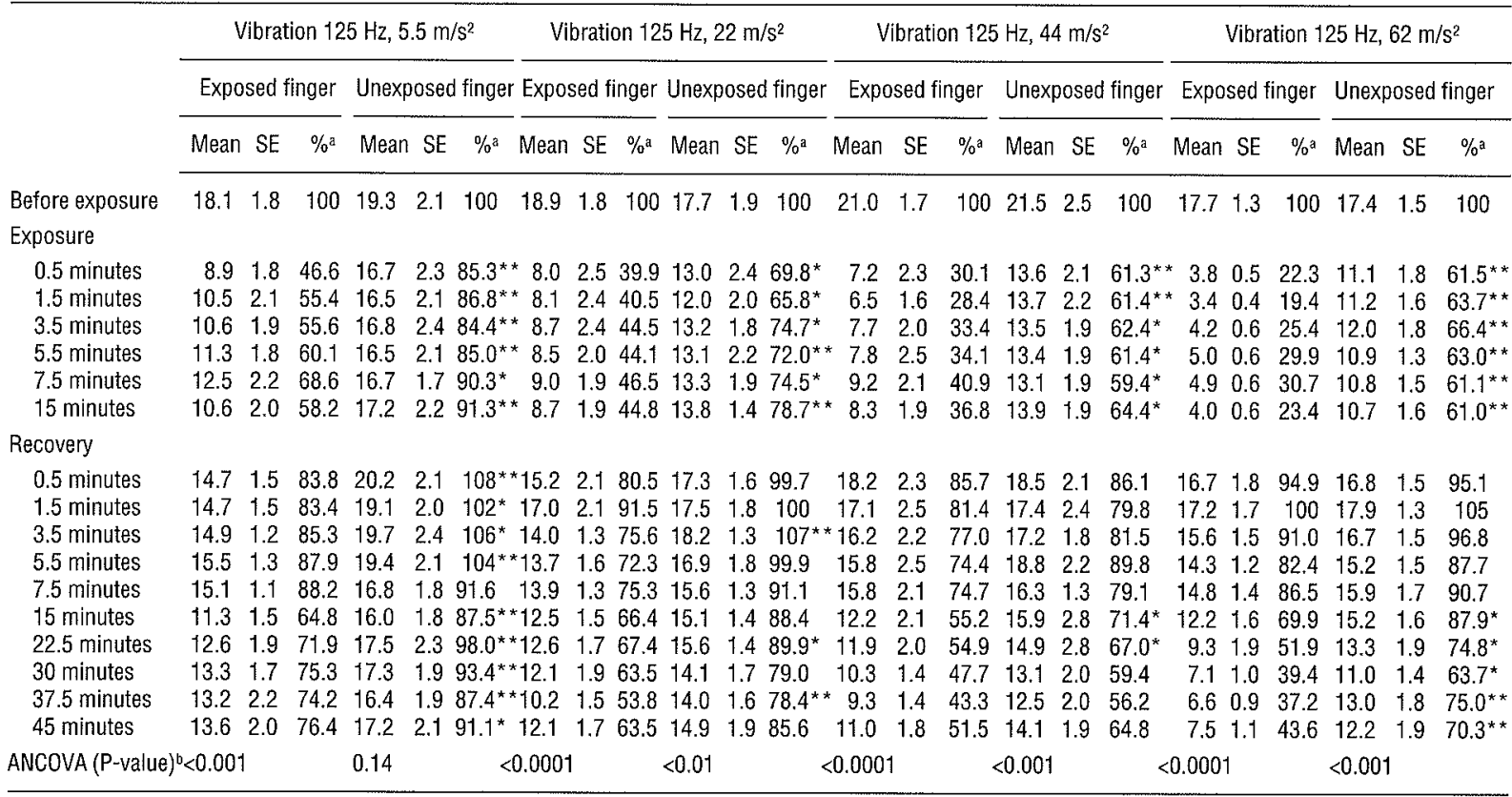

a Percentages of values before exposure.

- P-values of ANCOVA adjusted by age, finger volume, and room temperature.

${ }^{*} \mathrm{P}<0.05,{ }^{*} \mathrm{P}<0.01$; paired t-test (exposed versus unexposed finger).

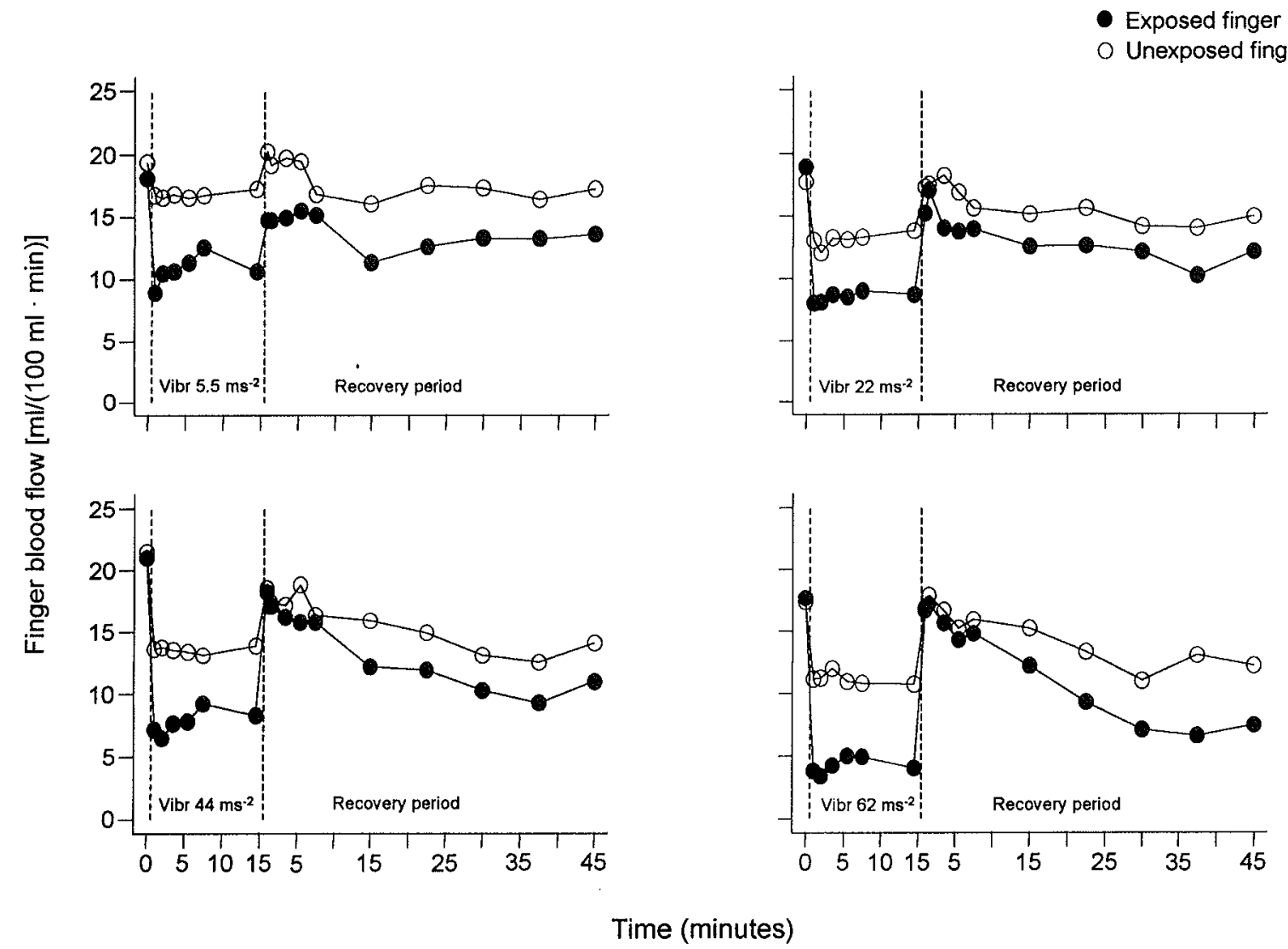

Figure 1. Mean values of finger blood flow measured in 10 healthy men before, during, and after 15-minute exposure to vibration with a frequency of $125 \mathrm{~Hz}$ and acceleration magnitudes of $5.5,22,44$, and $62 \mathrm{~m} / \mathrm{s}^{2}$ root-mean-square (rms). 
tude of $62 \mathrm{~m} / \mathrm{s}^{2}$ for the nonvibrated finger ( $\left.\mathrm{P}=0.032\right)$. The immediate postexposure $\mathrm{FBF}$, expressed as a percentage of the preexposure $\mathrm{FBF}$, did not differ among the $4 \mathrm{vi}$ bration magnitudes in either finger.

During the recovery period, the vibrated finger showed a decrease in FBF, compared with the preexposure values, about 15 to 30 minutes after the end of exposure to vibration magnitudes of 22 to $62 \mathrm{~m} / \mathrm{s}^{2}(P<0.05)$. A similar reduction in FBF was observed in the nonvibrated finger after exposure to acceleration magnitudes of 44 and $62 \mathrm{~m} / \mathrm{s}^{2}(\mathrm{P}<0.05)$. Likewise during the exposure period, digital vasoconstriction during recovery was stronger in the vibrated finger than in the nonvibrated finger. The Bonferroni test revealed that the changes in the FBF of the vibrated finger from 30 to 45 minutes during the recovery were greater after exposure to vibration magnitudes of either 44 or $62 \mathrm{~m} / \mathrm{s}^{2}$ than after exposure to vibration magnitudes of either 5.5 or $22 \mathrm{~m} / \mathrm{s}^{2}(\mathrm{P}<0.05)$.

\section{Discussion}

\section{Circulatory effects of acute vibration}

The results of this study are consistent with the findings of previous investigations indicating that acute exposure to unilateral vibration with a frequency of $125 \mathrm{~Hz}$ can produce hemodynamic changes in the ipsilateral and contralateral fingers of healthy subjects and vibration-exposed workers with or without vascular disorders $(1,4$, $5,9-11)$. The effects of vibration applied to a finger may not be restricted to the finger in that the vibration will be transmitted from the fingers to the hand and arm in a manner dependent on the frequency of the vibration. For the frequency of vibration used in this study, the effects of the vibration would be expected to have been in the fingers and of much reduced importance in the hand and arm.

The present study showed that acute exposures to $125-\mathrm{Hz}$ vibration of any magnitude in the range between 5.5 and $62 \mathrm{~m} / \mathrm{s}^{2}$ rms caused a reduction in the FBF of the vibrated finger, and this reduction was significant when the comparison was made with the preexposure measures. However, the extent of the response of the finger circulation to acute vibration was found to be dependent on the magnitude of the vibratory stimulus. Higher magnitudes of vibration at $125 \mathrm{~Hz}$ induced more powerful vasoconstrictor effects in the digital vessels than lower magnitudes of acceleration at the same vibration frequency. These circulatory effects were observed in both the ipsilateral and the contralateral finger, but they were more pronounced in the vibrated finger.

\section{Comparison to previous studies}

The findings of this investigation are consistent with those of a few previous experimental studies on the effects of the magnitude of hand-transmitted vibration on finger circulation $(9,11,12)$, even though these studies have some limitations associated with exposure conditions, experimental design, or the use of qualitative indicators of finger circulation.

On studying the digital circulatory effects of handtransmitted vibration on lumberjacks with and without VWF by means of piezoelectric or photoelectric plethysmography, Pyykkö et al (9) found that vasospastic reactions were more frequently triggered by $125-\mathrm{Hz}$ vibration with a high amplitude (peak-to-peak displacement of $530 \mu \mathrm{m}$ ) than by vibration with lower amplitudes (160$265 \mu \mathrm{m})$. Welsh reported a progressive reduction of FBF in normal subjects as the magnitude of vibration displacement at $80 \mathrm{~Hz}$ increased from 0.125 to $1.0 \mathrm{~mm}$ (12). Egan et al (11) measured FBF and FST in 12 healthy male volunteers whose right hands were exposed to 2-minute vibration of low or high intensity from a pneumatic chisel attached to a plate. Consistent with the present study, they found no change in FST throughout either level of vibration exposure. Both low and high intensity vibration caused reductions in the FBF of the right (vibrated) and left (nonvibrated) fingers during the exposure period. Moreover, the exposure to high intensity vibration was followed by incomplete recovery of the preexposure FBF, which was more pronounced than that observed after the end of the exposure to low intensity vibration. Even though the magnitude of the vibration exposure was not precisely quantified in that study, the findings seem to be in agreement with our findings.

Our findings indicate that digital vasoconstriction during vibration exposure and vasomotor after effects during recovery are associated with the magnitude of acute exposures to hand-transmitted vibration. The GEE approach to repeated measures data showed that there was a significant relation between the maximum reduction of FBF during both the vibration exposure and the recovery period and the magnitude of vibration (figure 2). This effect was stronger in the vibrated finger than in the nonvibrated finger during both periods.

\section{Pathophysiological mechanisms}

The pathophysiological mechanisms underlying the acute effects of hand-transmitted vibration on finger circulation have not yet been fully clarified. An immediate digital vasoconstriction at the beginning of vibration exposure, as well as persisting vasomotor effects after vibration has ended, has been reported in several investigations of either experimental animals or the human hand $(1,4,5,11,13,14)$. The prompt reduction of FBF in the ipsilateral finger and the evidence of vibration-induced digital vasoconstriction in the contralateral finger support the hypothesis that vibration can elicit a central sympathetic reflex through the activation of the mechanoreceptors located in the tissues of the hand $(5,9-11,13)$. It 


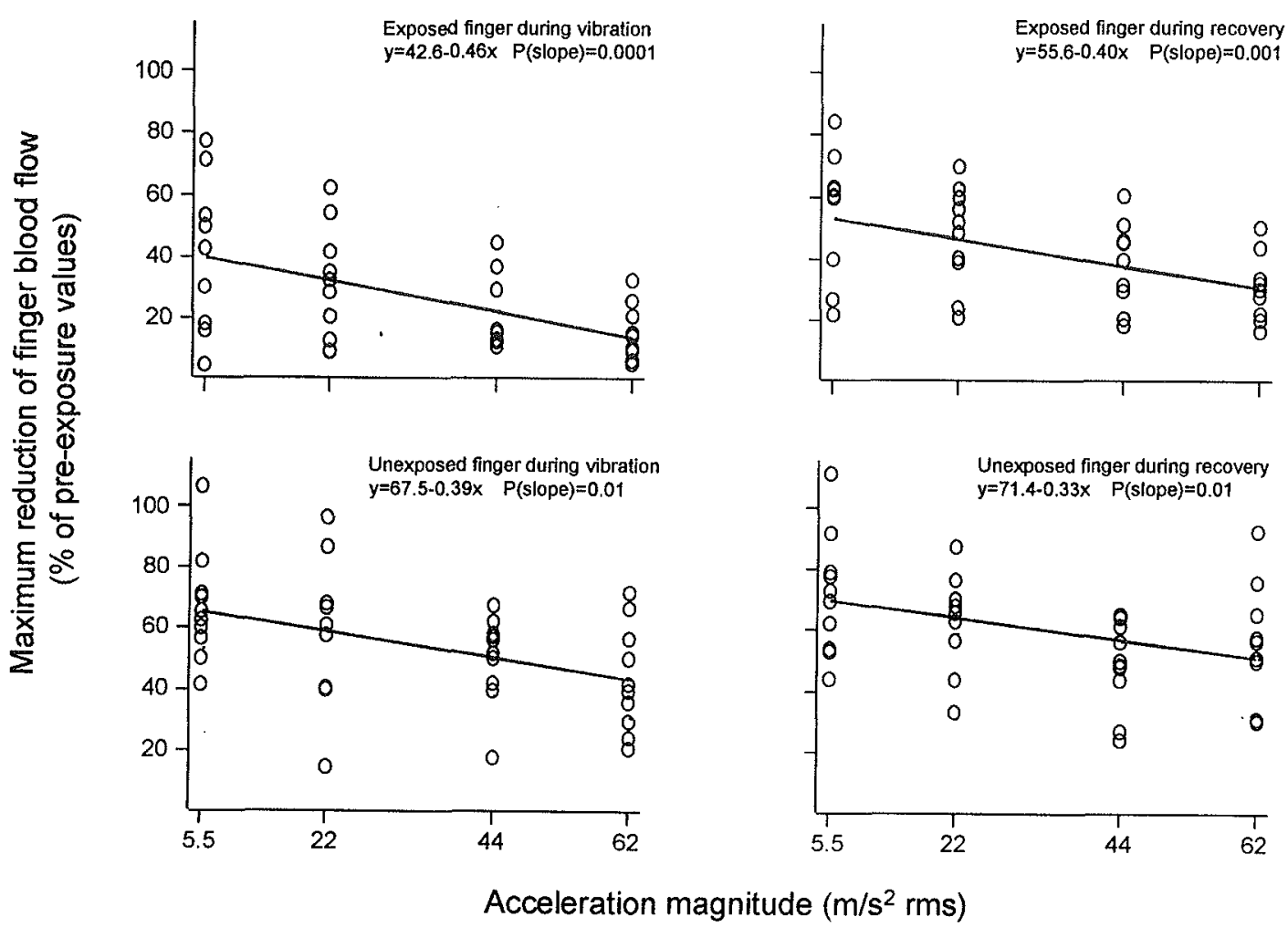

Figure 2. Relation between the maximum reduction of finger blood flow (expressed as the percentage of the preexposure values) during and after vibration exposure and the acceleration magnitude of the vibration.

is recognized that some of these mechanoreceptors, such as the Pacinian corpuscles, are connected with the sympathetic nervous system, and they respond to vibration stimuli in the frequency region between 63 and $500 \mathrm{~Hz}$ (15).

Local and neuroendocrine vasoregulatory factors have been considered by various researchers $(5,9,10$, 16) to play a role in the digital vasoconstriction induced by acute vibration. In the context of our investigation, it is worth noting that Azuma et al (14) found that the larger the vibration magnitude at frequencies above $30 \mathrm{~Hz}$, the stronger the responsiveness of femoral arterial strips of mongrel dogs to noradrenaline after vibration stimulation. This finding may suggest a conditioning effect of vibration on the arterial vessels, possibly by enhancing the constrictor response of the blood vessel wall to circulating vasopressor agents or substances released from the adrenergic nerves.

\section{Concluding remarks}

The results of this study indicate that acute exposure to $125-\mathrm{Hz}$ vibration can reduce $\mathrm{FBF}$ in vibrated and nonvibrated fingers and that the degree of the reduction in the finger circulation is related to the magnitude of the vibration exposure. This finding extends our previous observations in that, not only the duration of the exposure, but also the magnitude of vibration acceleration plays a role in the vasoconstrictor response of the digital vessels to acute vibration (1). The pattern of the hemodynamic changes induced by hand-transmitted vibration in both the exposed and the unexposed fingers was consistent with that observed in our previous investigations $(1,4$, 5), indicating that complex vasomotor mechanisms, mediated both centrally and locally (16), are involved in the reaction of digital vessels to acute vibration.

\section{References}

1. Bovenzi M, Lindsell CJ, Griffin MJ. Duration of acute exposures to vibration and finger circulation. Scand J Work Environ Health 1998;24:130-7.

2. International Organization for Standardization (ISO). Mechanical vibration - guidelines for the measurement and the assessment of human exposure to hand-transmitted vibration. Geneva: ISO, 1986. ISO 5349.

3. British Standards Institution (BSI). Measurement and evaluation of human exposure to vibration transmitted to the hand. London: BSI, 1987. BSI 6842

4. Bovenzi M, Griffin MJ, Ruffell CM. Acute effects of vibration on digital circulatory function in healthy men. Occup Environ Med 1995;52:834-41.

5. Bovenzi M, Griffin MJ. Haemodynamic changes in ipsilateral 
and contralateral fingers caused by acute exposures to handtransmitted vibration. Occup Environ Med 1997;54:566-76.

6. Greenfield ADM, Whitney RJ, Mowbray JF. Methods for the investigation of peripheral blood flow. Br Med Bull 1963;19:101-9.

7. Nielsen SL, Bell G, Lassen NA. Strain gauge studies of distal blood pressure in normal subjects and in patients with peripheral arterial disease: analysis of normal variation and reproducibility and comparison to intraartierial measurements. Scand J Clin Lab Invest 1973;31 suppl 128:103-9.

8. Diggle PJ, Liang KY, Zeger SL. Analysis of longitudinal data. Oxford: Oxford University Press, 1994. Oxford statistical science series, no 13.

9. Pyykkö I, Hyvärinen J, Färkillä M. Studies on the etiological mechanism of the vasospastic component of the vibration syndrome. In: Brammer AJ, Taylor W, editors. Vibration effects on the hand and arm in industry. New York (NY): Wiley \& Sons, 1982:13-24.

10. Greenstein D, Kester D. Acute vibration - its effect on digital blood flow by central and local mechanisms. In: Proc Inst Mech Eng [H] 1992;206:105—8.
11. Egan CE, Espie BH, McGrann S, McKenna KM, Allen JA. Acute effects of vibration on peripheral blood flow in healthy subjects. Occup Environ Med 1996;53:663—9.

12. Welsh $\mathrm{CL}$. The effect of vibration on digital blood flow, $\mathrm{Br} \mathbf{J}$ Surg 1980;67:708-10.

13. Olsen $N$. Vibration aftereffects on vasoconstrictor response to cold in the normal finger. Eur J Appl Physiol 1993;66:246-

14. Azuma T, Ohhashi T, Sakaguchi K. Vibration-induced hyperresponsiveness of arterial smooth muscle to adrenaline with special reference to Raynaud's phenomenon in vibration disease. Cardiovase Res 1978;12:758-64.

15. Santini M, Ibata Y, Pappas D. The fine structure of the sympathetic axon within the Pacinian corpuscle. Brain Res 1971;33:279-87.

16. Olsen N. Centrally and locally mediated vasomotor activities in Raynaud's phenomenon. Scand $\mathbf{J}$ Work Environ Health 1987;13:309-12.

Received for publication: 24 June 1998 